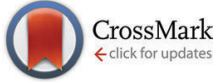

Cite this: J. Mater. Chem. B, 2016, 4, 6279

DOI: $10.1039 / c 6 t b 90121 b$

www.rsc.org/MaterialsB

\section{Correction: Anti-biofouling ability and cytocompatibility of the zwitterionic brushes-modified cellulose membrane}

\author{
Pingsheng Liu, ${ }^{* a b}$ Qiang Chen, ${ }^{\mathrm{bc}} \mathrm{Li} \mathrm{Li}^{\mathrm{a}}$ Sicong Lin ${ }^{\mathrm{b}}$ and Jian Shen ${ }^{\mathrm{ab}}$
}

Correction for 'Anti-biofouling ability and cytocompatibility of the zwitterionic brushes-modified cellulose membrane' by Pingsheng Liu et al., J. Mater. Chem. B, 2014, 2, 7222-7231.

The authors wish to notify readers that the experimental sections of this paper headed "Encapsulation of cells in zwitterionic hydrogels vs. PEGMA hydrogels" and "Live/dead staining of the cells encapsulated in the hydrogels" on page 7224-7225 associated with Fig. 10, overlap with sections previously published in the paper 'A comparative study of zwitterionic ligandsmediated mineralization and the potential of mineralized zwitterionic matrices for bone tissue engineering', J. Mater. Chem. B, 2014, DOI: 10.1039/C4TB01046A by a University of Massachusetts Medical School (USA) research team led by Professor Jie Song. Prof. Jian Shen was not aware of this previous publication. Further to this, the authors acknowledge that the sections of this work detailed above, including data presented in Fig. 10 and discussions associated with the cytocompatibility of zwitterioinic hydrogels, were funded by the US National Institutes of Health (R01AR055615), and were carried out by Pingsheng Liu at University of Massachusetts Medical School, USA, under the group leadership of Professor Jie Song.

The authors acknowledge that the experiments carried out by Pingsheng Liu regarding the examination of cytocompatibility using primary bone marrow stromal cells, including the mesh modification for this purpose, primary cell isolation as described in "Cytocompatibility of the CMs" (page 7224), Fig. 9, and related discussions in "Cytocompatibility assessment of the zwitterionic coatings" (page 7228-7229) were carried out at University of Massachusetts Medical School, USA, under the financial support of a grant from the Musculoskeletal Transplant Foundation and the group leadership of Professor Jie Song. Professor Jie Song did not have knowledge of the submission of this paper, and did not give her consent to the use of the above data.

The authors wish to notify readers that the remainder of the work in this paper took place as stated in the original manuscript, with financial support from the National High Technology Research and Development Program of China (2006AA03Z445), in Nanjing under the supervision of Professor Jian Shen and Senior Engineer Qiang Chen.

Pingsheng Liu, as the first author, wishes to clarify that his co-authors were not aware of the overlap detailed above or the potential conflict of the data ownership before the submission of the manuscript.

The authors apologise for any consequent inconvenience caused.

The Royal Society of Chemistry apologises for these errors and any consequent inconvenience to authors and readers.

\footnotetext{
a Jiangsu Key Laboratory of Biofunctional Materials, School of Chemistry and Materials Science, Nanjing Normal University, Nanjing 210097, People's Republic of China. E-mail: liupsh@gmail.com; Fax: +86 25 83599188; Tel: +86 2585891536

${ }^{b}$ School of Chemistry and Chemical Engineering, Nanjing University, Nanjing 210093, People’s Republic of China. E-mail: chem100@nju.edu.cn

${ }^{c}$ High Technology Research Institute of Nanjing University, Changzhou 213164, People's Republic of China
} 